\title{
Formation of Spherical Nanostructures by Controlled Aggregation of Gold Colloids
}

\author{
Irshad Hussain ${ }^{1,2}$, Zhenxin Wang ${ }^{1}$, Andrew I. Cooper ${ }^{1}$ and Mathias Brust ${ }^{1}$ \\ ${ }^{1}$ The University of Liverpool, Centre for Nanoscale Science, Department of Chemistry, \\ Crown Street, Liverpool, L69 7ZD. ${ }^{2}$ National Institute for Biotechnology and Genetic \\ Engineering (NIBGE), P.O. Box 577, Jhang Road, Faisalabad, Pakistan.
}

SUPPORTING INFORMATION

Page 1 of 3 


\begin{tabular}{|c|c|c|}
\hline $\begin{array}{c}\text { Final conc. of NDT in } \\
\text { solution }\end{array}$ & $\begin{array}{l}\text { Molar ratio of } \\
\text { NDT:GNP }=\mathbf{r}\end{array}$ & Observations \\
\hline $\begin{array}{c}16 \mathrm{nM} \\
32 \mathrm{nM} \\
50 \mathrm{nM} \\
67 \mathrm{nM} \\
83 \mathrm{nM} \\
167 \mathrm{nM} \\
833 \mathrm{nM} \\
1.3 \mu \mathrm{M} \\
1.7 \mu \mathrm{M} \\
2.0 \mu \mathrm{M} \\
2.3 \mu \mathrm{M} \\
2.7 \mu \mathrm{M} \\
3.0 \mu \mathrm{M} \\
3.3 \mu \mathrm{M} \\
3.7 \mu \mathrm{M}\end{array}$ & $\begin{array}{c}0.3 \\
0.6 \\
0.8 \\
1.1 \\
1.5 \\
3 \\
14 \\
22 \\
28 \\
33 \\
39 \\
44 \\
50 \\
55 \\
61 \\
\end{array}$ & $\begin{array}{l}\text { Intensity of the plasmon resonance } \\
\text { band of gold nanoparticles at } \\
526 \mathrm{~nm} \text { decreased gradually until it } \\
\text { approached its minimum value at } r \\
=61 \text {. At this value of } r \text {, gold } \\
\text { nanoparticles were almost } \\
\text { completely precipitated and the } \\
\text { supernatant was colourless. }\end{array}$ \\
\hline $\begin{array}{c}4.0 \mu \mathrm{M} \\
4.3 \mu \mathrm{M} \\
4.7 \mu \mathrm{M} \\
5.0 \mu \mathrm{M} \\
5.3 \mu \mathrm{M} \\
5.7 \mu \mathrm{M} \\
6.0 \mu \mathrm{M} \\
6.3 \mu \mathrm{M} \\
6.7 \mu \mathrm{M} \\
7.0 \mu \mathrm{M} \\
7.3 \mu \mathrm{M} \\
7.7 \mu \mathrm{M} \\
8.0 \mu \mathrm{M} \\
8.3 \mu \mathrm{M} \\
9.2 \mu \mathrm{M} \\
10.0 \mu \mathrm{M} \\
13.3 \mu \mathrm{M} \\
16.7 \mu \mathrm{M} \\
83.3 \mu \mathrm{M} \\
833 \mu \mathrm{M}\end{array}$ & $\begin{array}{c}67 \\
72 \\
78 \\
83 \\
89 \\
94 \\
100 \\
106 \\
111 \\
117 \\
122 \\
128 \\
133 \\
140 \\
150 \\
170 \\
220 \\
280 \\
1400 \\
14000 \\
\end{array}$ & $\begin{array}{l}\text { Intensity of the plasmon resonance } \\
\text { band increased gradually after the } \\
\text { value of } r \text { was increased beyond } 61 \text {. } \\
\text { It approached its maximum value at } \\
r=106 \text {, and was red shifted at } 546 \\
\text { nm. The colour of solution of these } \\
\text { assemblies was purple. There was } \\
\text { no significant change in the colour } \\
\text { or UV-visible spectra up to } r= \\
14000\end{array}$ \\
\hline $\begin{array}{l}1.7 \mathrm{mM} \\
3.3 \mathrm{mM}\end{array}$ & $\begin{array}{l}28000 \\
56000\end{array}$ & $\begin{array}{c}\text { Gold nanoparticles precipitated } \\
\text { again at } r \geq 28000 \text {. }\end{array}$ \\
\hline
\end{tabular}

Table 1: A summary of the reactant concentrations used in the high throughput screening experiment to form spherical assemblies of gold nanoparticles, and general observations. 


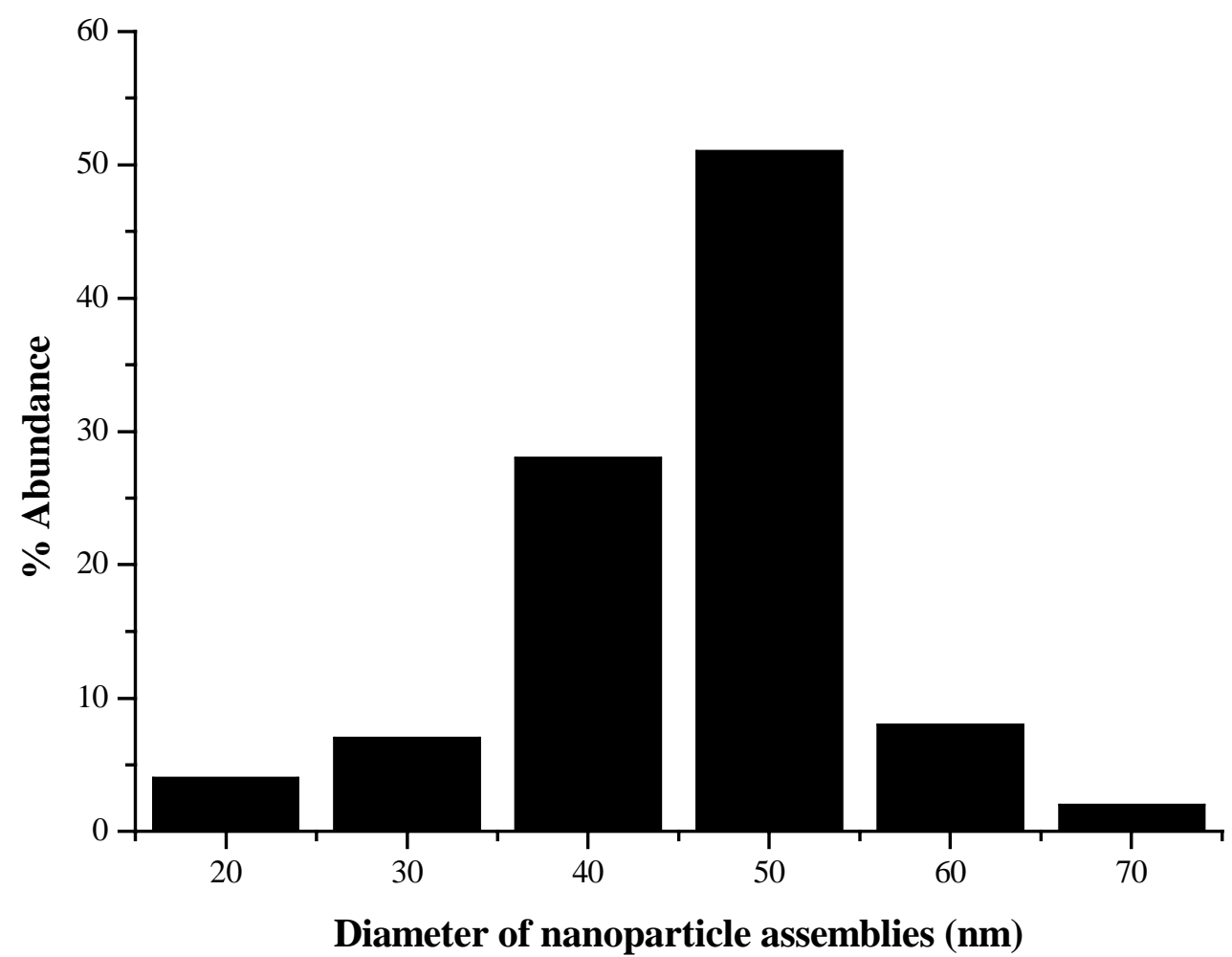

Figure 1: A histogram of the size of gold nanoparticle assemblies calculated from TEM measurements. 